\title{
Zur Erkennung von Wasserstoffsuperoxyd in der Milch.
}

\author{
Von \\ E. Heder. \\ Mitteilung aus dem Chemischen Untersuchungamte der Stadt Aachen.
}

Neuerdings wird vielfach als Milchkonservierungsmittel Wasserstoffsuperoxyd empfohlen und auch angewendet. Es soll vor anderen Frischhaltungsmitteln grobe Vorzüge besitzen, vor allem den, daß es während der Konservierung zerfällt und so unschädlich wird. ${ }^{1}$ ).

Der Nahrungsmittelchemiker wird jedoch die Verwendung von Wasserstoffsuperoxyd als Konservierungsmittel vorläufig als nicht erlaubt ansehen müssen; es ist deshalb wohl von Wert, eine einfache Reaktion zu besitzen, welche das Vorhandensein von selbst sehr geringen Mengen Wasserstoffsuperoxyd in der Milch anzeigt.

Fritzmann ${ }^{2}$ ) beobachtete zuerst die Blauviolettfärbung, welche beim Mischen von gewässerter schwach nitrathaltiger Milch mit Schwefelsäure in den beim Gerber'schen Fettbestimmungsverfahren vorgeschriebenen Mengenverhältnissen bei Anwesenheit von Spuren Formaldehyd entsteht. Er schlug die Verwertung dieser Erscheinung als Reaktion auf Salpetersäure in Milch vor. Allgemeineren Eingang hat dann das Verfahren in der von Gerber und $\mathrm{Wieske}^{3}$ ) vorgeschlagenen Form gefunden; die mit wenig Formaldehyd versetzte konzentrierte Schwefelsäure, als „Nitratreagens" vorrätig gehalten, wird zu gleichen Teilen mit der zu untersuchenden Milch gemischt. Bei Anwesenheit von Salpetersäure entsteht Blauviolettfärbung. Diese Reaktion ist nun jedoch keineswegs stets eindeutig.

K. Farnsteine ${ }^{4}$ ) fand, daß die Hehner'sche Reaktion auf Formaldehyd mit völlig reiner Schwefelsäure nicht eintritt, daß sie sich jedoch bei Gegenwart einer Spur Eisenchlorid einstellt. Ähnlich wirken Platinchlorid, weniger Quecksilberchlorid und Kaliumpermanganat. Der Eintritt der Reaktion ist also an das Vorhandensein eines Oxydationsmittels gebunden. Diese Tatsache ist auch erst kürzlich von Rosenheims) wieder hervorgehoben worden. Wie Salpetersäure wirken nun eine ganze Reihe anderer Oxydationsmittel. Von diesen kommt bei Milch noch das etwaige Vorhandensein von Wasserstoffsuperoxyd in Betracht. In der Tat entstehen beim Mischen von "Nitratreagens" mit Wasserstoffsuperoxyd enthaltender Milch violette Färbungen, deren Reinheit und Stärke von der Menge des zugesetzten Wasserstoffsuperoxyds abhängig ist. Bei einem Gehalt von $0,03 \%$ Wasserstoffsuperoxyd zeigt die Milch bei der Reaktion nur eine schmutzig violette Färbung; dagegen scheint letztere, wenn sie durch Nitrate hervorgerufen wird, reiner blauviolett zu sein. Jedoch ist es sicher

1) Das ist, wie A. Ren ard hervorhebt und wie ich auf Grund von gleich mitzuteilenden Versuchen bestätigen kann, aber nur bis zu einem gewissen Grade richtig. Sobald nämlich die Konzentration des Wasserstoffsuperoxyds in der Milch eine gewisse Grenze erreicht hat, bleiben erhebliche Mengen des Konservierungsmittels noch nach langer Zeit nachweisbar.

2) Zeitschr. offentl. Chem. 1897, 3, 23; J. K onig, Untersuchung landwirtschaftlich und gewerblich wichtiger Stoffe, 3. Aufl, 1906, 8, 486.

3) Molkerei-Ztg. Berlin 1902, 12,61; J. König, Untersuchung landwirtschaftlich and gewerblich wichtiger Stoffe, 3. Aufl. 1906, S. 486.

*) Forschangsherichte über Lebensmittel etc. $1896, \mathbf{3}, 363$.

5) Analyst 1907, 32, 108; Chem.-Ztg. 1907, 31, 374. 
zweckmäßig, vor dem Nachweis von Salpetersäure mit "Nitratreagens" sich vorher von der Abwesenheit von Wasserstoffsuperoxyd zu überzeugen.

Eine weit schönere und empfindlichere Reaktion kann man mit schwach wasserstoffsuperoxydhaltiger Milch erzielen, wenn man diese unter Zusatz von einem Tropfen schwacher Formalinlösung mit einem gleichen Volumen konzentrierter Salzsäure $(1,19)$ behandelt. Es entstehen dábei prachtvolle blauviolette Färbungen. Bei einigermaßen beträchtlichem Zusatze genügt schon die beim Mischen gleicher Volumen Milch und konzentrierter Salzsäure hervorgerufene Temperaturerhöhung $\left(30-35^{\circ}\right)$, um die Reaktion auszulösen. Beim Kochen der Mischung tritt sie sofort ein. Jedoch bilden sich auch beim Kochen von reiner Milch mit konzentrierter Salzsäure leicht schmutzig violette Färbungen, welche nach C. Amthor $\mathrm{r}^{1}$ ) die Folge einer Reaktion zwischen den Eiweißkörpern und dem Zucker der Milch sind. Diese Miß̈färbungen treten bei $60^{\circ}$ noch nicht ein; andererseits genügt diese Temperatur, um den Nachweis von Wasserstoffsuperoxyd in Milch mit formalinhaltiger Salzsäure in ganz kurzer Zeit zu ermöglichen. Am besten stellt man einfach das Reagensrohr mit der Mischung $(5 \mathrm{ccm}$ Milch $+5 \mathrm{ccm}$ Salzsäure unter Zusatz von einem Tropfen schwacher Formalinlösung) in ein Bad von etwa $60^{\circ}$ und beobachtet kurze Zeit (höchstens 3-4 Minuten), wobei zweckmäßig einige Male umgeschüttelt wird.

Der Zusatz von 0,01\% Wasserstoffsuperoxyd verursacht in ganz kurzer Zeit eine sehr schöne Reaktion; auch bei einem Gehalt von $0,006 \%$ Wasserstoffsuperoxyd trat dieselbe noch deutlich ein. Eine $0,003 \%$ enthaltende Milch gab nurmehr eine schwache Violettfärbung. Milch, welche frei von Wasserstoffsuperoxyd ist, gibt bei der geschilderten Behandlung allmählich (nach mehreren Minuten) eine Gelbfärbung.

Frische Milch wurde in verschiedenen Mengenverhältnissen mit Wasserstoffsuperoxyd versetzt und in mehreren $Z$ wischenräumen auf den etwa noch vorhandenen Gehalt an Konservierungsmittel nach dem beschriebenen Verfahren untersucht.

Die Reaktion trat ein:

\begin{tabular}{|c|c|c|c|c|c|}
\hline Bei Zusatz von & $\begin{array}{l}\text { Sofort nach } \\
\text { dem Znsatz }\end{array}$ & $\begin{array}{c}\text { Nach } \\
8 \text { Stunden }\end{array}$ & $\begin{array}{l}\text { Nach } \\
22 \text { Stunden }\end{array}$ & $\begin{array}{l}\text { Nach } \\
48 \text { Stunden }\end{array}$ & $\begin{array}{c}\text { Nach } \\
72 \text { Stunden }\end{array}$ \\
\hline $\begin{array}{ll}0,035 \% & \mathrm{H}_{2} \mathrm{O}_{2} \\
0,075 \% & * \\
0,150 \% & " \\
0,300 \% & *\end{array}$ & $\begin{array}{l}\text { stark } \\
" ~ \\
" \\
"\end{array}$ & $\begin{array}{c}\text { modentlich } \\
\text { stark } \\
n\end{array}$ & $\begin{array}{c}\text { undeatlich } \\
\text { schwach } \\
\text { stark. } \\
*\end{array}$ & $\begin{array}{c}\text { nicht } \\
\text { undeutlich } \\
\text { stark } \\
n\end{array}$ & $\begin{array}{l}\text { nicht } \\
\text { stark } \\
\text { " }\end{array}$ \\
\hline
\end{tabular}

Diese Befunde bestätigen in wesentlichen die Beobachtungen, welche A. Renard ${ }^{2}$ ) gemacht hat. Wenn der Wasserstoffsuperoxyd-Gehalt der Milch $0,06^{\circ} \%$ nicht übersteigt, so wird das Wasserstoffsuperoxyd in relativ kurzer Zeit in Wasser und Sauer stoff zerlegt. Mit erböhtem Zusatz tritt das Ende der Zersetzung immer langsamer ein; „sie hört bei einem Zusatz von $0,15 \%$ auf, jemals eine vollständige zu werden".

Jedoch ist auch ein Zusatz von $0,075 \%$ noch am folgenden Tage nachzuweisen.

Nun wird auch die vorgeschlagene Reaktion noch durch andere Oxydationsmittel als durch Wasserstoffsuperoxyd hervorgerufen, so durch Platinchlorid, Eisen-

1) Diese Zeitschrift 1900, 3, 1105.

2) Jahresbericht über die Fortschritte in der Untersuchung der Nahrungs- und Genußmittel 1905, S. 40. 
chlorid, Kupfersulfat, und für don etwaigen Nachweis in Milch kommen in Frage Salpetersäure und salpetrige Säure. Salpetersäure ruft jedoch die Reaktion unter den vorgeschlagenen Bedingungen nicht hervor, während beim Kochen der Mischung eine schmutzig violette Färbung eintritt. Wohl aber wird die Reaktion wie auch E. Vo i s en et ${ }^{1}$ ) zeigt, schnell hervorgerufen durch Spuren salpetriger Säure und zwar genügt auch hier meist die durch das Mischen der Milch mit der konzentrierten Salzsäure verursachte Temperaturerhöhung. Als untere Grenze der Empfindlichkeit wurde für die Reaktion ein Gehalt von $0,5 \mathrm{mg} \mathrm{N} \mathrm{N}_{2} \mathrm{O}_{3}$ in $100 \mathrm{~cm}$ Milch festgestellt. Bei einem Gehalt von $0,25 \mathrm{mg}$ in $100 \mathrm{ccm}$ war keine Färbung mehr zu beobachten. Nimmt man nun den Fall an, daß ein zur Wässerung von Milch dienendes Brunnenwasser den erheblichen Gehalt von $20 \mathrm{mg} \mathrm{N} \mathrm{N}_{2} \mathrm{O}_{3}$ im Liter habe, so würde es einer Mindestwässerung von etwa $20 \%$ bedürfen, um den Eintritt der Reaktion durch salpetrige Säure hervorzurufen.

Aus dem Gesagten erhellt, daß in nichtgewässerter Milch der Nachweis von Wasserstoffsuperoxyd auf die geschilderte einfache Weise schnell zu führen ist. Ist die Milch erheblich gewässert, so hat man sich bei einem positiven Ausfall der Reaktion auf andere Weise von der Abwesenheit von salpetriger Säure zu überzeugen.

1) Bull. Soc. Chim. Paris 1905, [3] 33, 1198; Chem. Zentralbl. 1906, I, 90.

\section{Referate.}

\section{Fleisch, Fleischwaren und diätetische Nährmittel.}

H. S. Grindley und H. S. Woods: Die Chemie des Fleisches. Verfahren zur Bestimmung von Kreatin und Kreatinin in Fleisch und Fleischpräparaten. (Journ. of biolog. Chem. 1907, 2, No. 4. Sonderabdruck) - Im Anschluf an die früheren Arbeiten (Z. 1904, 8, 741; 1906, 11, 280; 1906, 12, 352 und 556) haben die Verff. versucht, die Mengen der verschiedenen wasserlöslichen Teile des Fleisches zu bestimmen, in erster Linie des Kreatins und Kreatinins. Sie benutzen hierzu mit Erfolg das kolorimetrische Verfahren von Folin (Zeitschr. physiol. Chem. 1904, 12, 223) zur Bestimmung dieser Körper im Urin. Bei den Versuchen, die Mengen des Kreatinins aus wässerigen Extrakten von frischem Fleiseh zu ermitteln, wurde keine Spur von Kreatinin erhalten. Die alkalischen Pikratlösungen färbten sich beim Stehen allmählich rot, ein Beweis dafür, daß die vorhandene natürliche Säure beim Eindampfen der Lösung das Kreatin in Kreatinin umwandelt. Zur Bestimmung des Kreatins verfahren die Verff. folgendermaßen : $500 \mathrm{ccm}$ des in früher (Z. 1906, 11, 280) beschriebener Weise bereiteten wässerigen Fleischauszuges werden auf $50 \mathrm{com}$ eingedampft; die koagulierten Eiweißstoffe werden abfiltriert und mit heißem Wasser gut ausgewaschen. Zum Filtrat gibt man dann $25 \mathrm{ccm} 1 / 10$ N.Salzsäure und dampft es auf $10-15 \mathrm{ccm}$ ein, setzt $50 \mathrm{ccm}$ Wasser und noch einmal $10 \mathrm{ccm}$ Salzsäure hinzu und dampft abermals auf $10-15 \mathrm{ccm}$ ein. Es ist nämlich von Wichtigkeit, alles Kreatin in Kreatinin überzuführen. Nach der Ablühlung bringt man die Lösung in ein $100 \mathrm{ccm}-$ Maßkölbchen und füllt bis zur Marke auf. Von dieser Lösung bringt man einen, $200 \mathrm{ccm}$ des ursprünglichen wässerigen Auszuges entsprechenden, aliquoten Teil in einen $500 \mathrm{ccm}-\mathrm{Kolben}$, setzt $15 \mathrm{ccm}$ einer gesättigten $(1,2 \%$-igen) Pikrinsäurelösung und $5 \mathrm{~cm} 10 \%$-ige Natronlauge hinzu, schüttelt tüchtig und läßt 5 Minuten lang stehen. Dann füllt man die Lösung zu $500 \mathrm{ccm}$ auf, mischt gut und vergleicht sie in einem D ubos c q'schen Kolorimeter mit 8,0 mm einer $1 / 2$ N.-Kaliumbichromatlösung. Der Genauigkeit halber mache man mehrere 